\title{
THE POSITION OF THE RADICAL IN AN ALGEBRA
}

\author{
BY
}

\section{MARSHALL HALL}

1. Introduction. The papers of Brauer, Nesbitt, and Nakayama( $\left.{ }^{1}\right)$ have given a great deal of information about algebras with a radical. These cover wide and interesting, but nevertheless special, classes of algebras. This paper gives a new approach to the study of the most general class of algebras with a radical, starting from the fundamental theorem that every linear associative algebra is uniquely decomposable as the direct sum of a semisimple algebra and an algebra bound to its radical. Here an algebra is said to be bound to its radical (for short: a bound algebra) if the two-sided annihilators of the radical are contained in the radical. In the light of this result, further investigations on algebras with a radical may be confined to bound algebras. A bound algebra is largely determined by its radical. In particular (Theorem 3.8) if the radical is of order $s$, the bound algebra is at most of order $s^{2}+s+1$.

A combination of the right and left representations of a bound algebra on its radical yields a faithful representation of the algebra modulo the twosided annihilator of the radical. To obtain a faithful representation of the bound algebra itself (Theorem 3.2) we must adjoin to this representation a system of "remnants" comparable to the factor sets used in the extension of groups or in the theory of normal simple algebras.

A bound algebra is composed (Theorem 3.6) of its radical combined with three orthogonal algebras of which two are semisimple. The third has a unit and is "doubly represented."

The final section of this paper is concerned with the problem of constructing all algebras with a given radical. Some examples are given to illustrate different aspects of this problem.

2. Decomposition of algebras. Let $\mathfrak{A}$ be an arbitrary linear associative algebra and let $\Re$ be its radical.

Presented to the Society, April 7, 1939; received by the editors March 7, 1940. This paper was received by the editors of the Annals of Mathematics June 19, 1939, accepted by them, and later transferred to these Transactions.

(1) R. Brauer, C. Nesbitt, On the regular representations of algebras, Proceedings of the National Academy of Sciences, vol. 23 (1937); On the Modular Representation of Groups of Finite Order, University of Toronto Studies, Mathematical Series, vol. 4, 1937.

T. Nakayama, C. Nesbitt, Note on symmetric algebras, Annals of Mathematics, (2), vol. 39 (1938).

C. Nesbitt, On the regular representations of algebras, Annals of Mathematics, (2), vol. 39 (1938).

T. Nakayama, Some studies on regular representations, induced representations, and modular representations, Annals of Mathematics, (2), vol. 39 (1938); On Frobeniusean algebras I, ibid., vol. 40 (1939); On Frobeniusean algebras II, to appear shortly; On the structure of symmetric algebras and Galois moduli over modular fields, to appear shortly. 
TheOREm 2.1. If $\mathfrak{a}$ is a left ideal of $\mathfrak{A}$, there is an idempotent e such that $\mathfrak{a}=(e)_{l}+\mathfrak{r}_{1}$ where $\mathfrak{r}_{1} \subset \Re$ and $\mathfrak{r}_{1} e=0$. If $\mathfrak{b}$ is a right ideal, there is an idempotent $f$ such that $\mathfrak{b}=(f)_{r}+\mathfrak{r}_{2}$ where $\mathfrak{r}_{2} \subset \Re$ and $f_{\mathfrak{r}_{2}}=0$. If $\mathfrak{c}$ is a two-sided ideal, there is an idempotent $g$ such that $\mathrm{c}=(g)_{l}+\mathfrak{r}_{3}=(g)_{r}+\mathfrak{r}_{4}$ where $\mathfrak{r}_{3}, \mathfrak{r}_{4} \subset \Re$ and $\mathfrak{r}_{3} g=g \mathfrak{r}_{4}$ $=0$.

For the right or left ideals this theorem has been proved by the author $\left({ }^{2}\right)$. A two-sided ideal $c$ may be considered as both a left ideal and a right ideal:

$$
\begin{array}{lll}
\mathfrak{c}=(e)_{l}+\mathfrak{r}_{1}, & \mathfrak{r}_{1} e=0, & \mathfrak{r}_{1} \subset \Re, \\
\mathfrak{c}=(f)_{r}+\mathfrak{r}_{2}, & f \mathfrak{r}_{2}=0, & \mathfrak{r}_{2} \subset \Re .
\end{array}
$$

Here $f=w e+r_{1}$ where $r_{1} e=0$ and $e=f u+r_{2}$ where $f r_{2}=0$. Hence $f e=w e=f u$ and $f-e=r_{1}-r_{2} \varepsilon \Re$. If $x \varepsilon(f)_{r}+\mathfrak{r}_{2}=\mathfrak{c}, x=f v+s, s \varepsilon(\Re \cap \mathrm{c})$. Hence $x=(e+r) v$ $+s=e v+r v+s$ where $r v+s \varepsilon(\Re \cap c)$, and so $x \varepsilon(e)_{r} \cup(\Re \cap c)$. But since $e \boldsymbol{\varepsilon} \mathfrak{c},(e)_{r} \subset \mathfrak{c}$, whence $\mathfrak{c} \subset(e)_{r} \cup(\Re \cap \mathfrak{c}) \subset \mathfrak{c}$ or $\mathfrak{c}=(e)_{r} \cup(\Re \cup \mathfrak{c})$. But $(\Re \cap \mathfrak{c})$ $=e(\Re \cap \mathfrak{c})+\mathfrak{r}_{3}$ where $e \mathfrak{r}_{3}=0, \mathfrak{r}_{3} \subset \Re$. Hence $\mathfrak{c}=(e)_{r} \cup\left(e(\Re \cap \mathfrak{c})+\mathfrak{r}_{3}\right)=(e)_{r}+\mathfrak{r}_{3}$. Thus in the two representations of (2.1) we may assume without loss of generality that the idempotents $e$ and $f$ are the same, which is the statement of the theorem.

Definition. An algebra $\mathfrak{A}$ is said to be bound to its radical $\mathfrak{R}$ (briefly: $\mathfrak{A}$ is a bound algebra) if for $c \boldsymbol{\varepsilon} \mathfrak{A}, c \Re=\Re c=0$ implies that $c \boldsymbol{\varepsilon} \Re$.

Theorem 2.2. Any linear associative algebra is uniquely decomposable as the direct sum of a semisimple algebra and a bound algebra.

Let $\mathfrak{A}$ be a linear associative algebra, and let $\Re$ be its radical. The twosided annihilators of $\Re$, elements $c$ such that $c \Re=\Re c=0$, form a two-sided ideal $\Re^{t}$. By Theorem 2.1 there is an idempotent $g$ such that $\Re^{t}=(g)_{l}+r_{3}$ $=(g)_{r}+\mathfrak{r}_{4}$ where $\mathfrak{r}_{3}, \mathfrak{r}_{4} \subset \Re, \mathfrak{r}_{3} g=0, g \mathfrak{r}_{4}=0$. If

$$
\mathfrak{A}=\mathfrak{A}_{1}+\mathfrak{A}_{2}+\mathfrak{A}_{3}+\mathfrak{A}_{4}
$$

is the two-sided Pierce decomposition of $\mathfrak{A}$ with respect to $g$, we have, for $a_{i} \varepsilon \mathfrak{A}_{i}$,

$$
\begin{array}{llll}
g a_{1}=a_{1}, & g a_{2}=a_{2}, & g a_{3}=0, & g a_{4}=0, \\
a_{1} g=a_{1}, & a_{2} g=0, & a_{3} g=a_{3}, & a_{4} g=0 .
\end{array}
$$

Consider any $a_{2}$. $\Re a_{2}=\Re g a_{2}=0 \cdot a_{2}=0$. Also $a_{2} \Re=g a_{2} \Re=g\left(a_{2} \Re\right) \subset g \Re=0$. Hence $a_{2} \varepsilon \Re^{t}$, and so $a_{2}=w g+r$ where $r \varepsilon \Re, r g=0$. Here $0=a_{2} g=w g$ and $a_{2}=r \varepsilon \Re$. Hence $a_{2}=g a_{2}=g r \varepsilon g \Re=0$ and $a_{2}=0$. Hence $\mathfrak{A}_{2}=0$. Similarly $\mathfrak{A}_{3}=0$. Hence (2.2) reduces to

(2) A type of algebraic closure, Annals of Mathematics, (2), vol. 40 (1939), Theorem 6.1. It is evident that the use of a unit in this theorem is not essential. 


$$
\mathfrak{A}=\mathfrak{A}_{1} \oplus \mathfrak{A}_{4},
$$

where the sum is direct since in consequence of the relations (2.3) $\mathfrak{A}_{1} \mathfrak{A}_{4}=\mathfrak{A}_{4} \mathfrak{A}_{1}$ $=0$. Since also $\mathfrak{A}_{1}^{2} \subset \mathfrak{A}_{1}, \mathfrak{A}_{4}^{2} \subset \mathfrak{A}_{4}, \mathfrak{A}_{1}$ and $\mathfrak{A}_{4}$ are subalgebras of $\mathfrak{A}$. Moreover the elements annihilated by $g$ on both sides are in $\mathfrak{A}_{4}$, and so $\mathfrak{R} \subset \mathfrak{A}_{4}$. If $\mathfrak{A}_{1}$ had a radical it would be part of the radical of $\mathfrak{A}$, which is in $\mathfrak{A}_{4}$. Hence $\mathfrak{A}_{1}$ has no radical, and is semisimple. Moreover $\Re$ is the radical of $\mathfrak{A}_{4}$. Suppose $a_{4} \varepsilon \mathfrak{U}_{4}$ is a two-sided annihilator of $\Re$. Then $a_{4} \varepsilon(g)_{l}+\mathfrak{r}_{3}$ and $a_{4}=w g+r_{3}$ where $r_{3} g=0$. But $0=a_{4} g=w g$, and so $a_{4}=r_{3} \varepsilon \Re$. Hence the two-sided annihilators of $\Re$ in $\mathfrak{H}_{4}$ are in $\Re$, and $\mathfrak{A}_{4}$ is bound to its radical $\mathfrak{R}$. $\mathfrak{A}$ is the direct sum of the semisimple algebra $\mathfrak{A}_{1}$ and the bound algebra $\mathfrak{A}_{4}$.

Now suppose

$$
\mathfrak{A}=\mathfrak{B} \oplus \mathfrak{C}
$$

is any decomposition of $\mathfrak{A}$ as the direct sum of a semi-simple algebra $\mathfrak{B}$ and a bound algebra $\mathfrak{C}$. The unit $h$ of $\mathfrak{B}$ is a two-sided annihilator of $\mathbb{S}$ and a fortiori of $\Re \subset \mathfrak{C}$. Hence, under the decomposition (2.4) of $\mathfrak{A}, h=h_{1}+h_{4}$ and each of $h_{1}, h_{4}$ is a two-sided annihilator of $\Re$. But $h=h^{2}=h_{1}^{2}+h_{4}^{2}$, whence $h_{4}^{2}=h_{4}$. As $\mathfrak{A}_{4}$ is a bound algebra, $h_{4} \subset \Re$. An idempotent can be in the radical only if it is zero, and so $h_{4}=0, h=h_{1} \varepsilon \mathfrak{A}_{1}$ and $h=g h=h g$. A similar argument shows that $g=h g=g h$, starting from the decomposition of $g$ in (2.5). Combining results, we obtain $g=h$. Evidently (2.5) as a direct sum is the two-sided Pierce decomposition of $\mathfrak{A}$ with respect to $h$ and consequently must be identical with (2.4). This proves the uniqueness part of the theorem.

3. Representation and properties of bound algebras. Let $\mathfrak{A}$ be a bound algebra over a field $K$ and $x_{1}, \cdots, x_{s}$ a basis of its radical $\Re$. Then if $c$ is an arbitrary element of $\mathfrak{A}$,

$$
\begin{array}{rlrl}
x_{i} c & =\sum_{j=1}^{s} a_{i j} x_{j}, & i=1, \cdots, s, \\
c x_{i}=\sum_{j=1}^{s} b_{i j} x_{j}, & i=1, \cdots, s,
\end{array}
$$

and we have the right representation of $\mathfrak{A}{ }^{\circ}$ on $\mathfrak{R}$ :

$$
c \rightarrow\left(a_{i j}\right)=R(c)
$$

and the left representation $\left({ }^{3}\right)$ of $\mathfrak{A}$ on $\Re$ :

$$
c \rightarrow\left(b_{i j}\right)=L(c) .
$$

Now (3.2) is a faithful representation of $\mathfrak{A} / \Re^{r}$ since the $c$ 's mapped onto zero are the right annihilators of $\Re$. Similarly (3.3) is a faithful representation

$\left(^{3}\right)$ For the left representation $c d \rightarrow L(d) L(c)$. Ordinarily the transpose $L(c)^{T}$ is used to preserve the order of multiplication. But in this paper it seems desirable to leave the representation in this form. 
of $\mathfrak{A} / \Re^{l}$. (Note that $\Re^{r}$ and $\Re^{l}$ are both two-sided ideals.) A combination of (3.2) and (3.3) is better than either one separately. If we put

$$
c \rightarrow[R(c), L(c)]
$$

we have the rules of combination

$$
\begin{aligned}
c_{1}+c_{2} & \rightarrow\left[R\left(c_{1}\right)+R\left(c_{2}\right), L\left(c_{1}\right)+L\left(c_{2}\right)\right], \\
c_{1} c_{2} & \rightarrow\left[R\left(c_{1}\right) R\left(c_{2}\right), L\left(c_{2}\right) L\left(c_{1}\right)\right], \\
k c & \rightarrow[k R(c), k L(c)],
\end{aligned}
$$

Here (3.4) subject to the combinatory rules (3.5) is a faithful representation of $\mathfrak{A}$ modulo $\mathfrak{R}^{t}$.

TheOREM 3.1. In the representation (3.4) every matrix $R\left(c_{1}\right)$ permutes with every matrix $L\left(c_{2}\right)$.

This well known theorem on representations is an immediate consequence of the associative law $c_{2}\left(\Re c_{1}\right)=\left(c_{2} \Re\right) c_{1}$.

To obtain a faithful representation of $\mathfrak{A}$ we must extend the representation (3.4) by the adjunction of $\Re^{t}$. Let $z_{1}, \cdots, z_{q}$ be a basis of $\Re^{t}$ and extend this to a basis of $\Re, \bar{u}_{1}, \cdots, \bar{u}_{p}, z_{1}, \cdots, z_{q}$, where $p+q=s$ and finally to a basis of $\mathfrak{A}, \bar{u}_{1}, \cdots, \bar{u}_{p}, \bar{u}_{p+1}, \cdots, \bar{u}_{m}, z_{1}, \cdots, z_{q}$. If, in the homomorphism $\mathfrak{A} \rightarrow \mathfrak{A} / \mathfrak{R}^{t}, \bar{u}_{i} \rightarrow u_{i}$, then $u_{1}, \cdots, u_{m}$ form a basis of $\mathfrak{A} / \mathfrak{R}^{t}$. We shall call $\bar{u}_{i}$ the representative of its class in $\mathfrak{A}$ modulo $\mathfrak{R}^{t}$. To extend this concept of representative, suppose an arbitrary $c \varepsilon \mathfrak{A}$ is given by

$$
c=\sum_{j=1}^{m} c_{j} \bar{u}_{j}+\sum_{k=1}^{q} r_{k} z_{k}
$$

Then the mapping $\mathfrak{A} \rightarrow \mathfrak{A} / \mathfrak{R}^{t}$, which we may suppose given by (3.4), maps $c$ onto an element $\gamma=\sum_{j=1}^{m} c_{j} u_{j}$. If we now write

$$
\bar{\gamma}=\sum_{j=1}^{m} c_{j} \bar{u}_{j},
$$

we call $\bar{\gamma}$ the representative of the class of $\mathfrak{A}$ modulo $\Re^{t}$ mapped onto $\gamma$. Hence any $c$ of $\mathfrak{A}$ is expressible uniquely as

$$
c=\bar{\gamma}+r \text {, }
$$

where $c \rightarrow \gamma$ by the homomorphism (3.4) and $r \varepsilon \Re^{t}$.

Let us now suppose the basis of $\Re$ used in (3.4) is chosen to be $\bar{u}_{1}, \cdots, \bar{u}_{p}$, $z_{1}, \cdots, z_{q}$. Since $\Re^{t}$ is a two-sided ideal, the matrices $R(c)$ and $L(c)$ must be of the form

$$
R(c)=\left(\frac{R_{11}(c)}{\cdot 0} \mid \frac{R_{12}(c)}{R_{22}(c)}\right), . \quad L(c)=\left(\frac{L_{11}(c)}{0} \mid \frac{L_{12}(c)}{L_{22}(c)}\right),
$$


where $R_{22}(c)$ and $L_{22}(c)$ are $q$ by $q$ matrices giving the right and left transformations induced on $\Re^{t}$ by $c$. If

$$
r=\sum_{k=1}^{q} r_{k} z_{k}
$$

is any element of $\Re^{t}$, then

$$
r c=\sum_{k=1}^{q} t_{k} z_{k}, \quad c r=\sum_{k=1}^{q} s_{k} z_{k},
$$

where

$$
\left(\begin{array}{c}
t_{1} \\
\dot{\vdots} \\
\dot{t}_{q}
\end{array}\right)=R_{22}(c)\left(\begin{array}{c}
r_{1} \\
\dot{\vdots} \\
\dot{r}_{q}
\end{array}\right), \quad\left(\begin{array}{c}
s_{1} \\
\vdots \\
\dot{s_{q}}
\end{array}\right)=L_{22}(c)\left(\begin{array}{c}
r_{1} \\
\dot{s} \\
r_{q}
\end{array}\right) .
$$

TheOREM 3.2. RePRESENTATION OF Bound ALGEBRAs. Let $\mathfrak{A}$ be an algebra bound to its radical $\mathfrak{R}$. Then a faithful representation of $\mathfrak{A}$ is given by

$$
c \rightleftarrows[R(c), L(c), W(c)],
$$

where $R(c), L(c)$ are given by (3.1)-(3.3) and are of the type (3.9) and $W(c)$ $=\left(r_{1} \ldots r_{q}\right)^{\prime}$ (the prime indicating the transpose of the vector) is determined by $r=\sum_{k=1}^{a} r_{k} z_{k}$ in (3.6). The rules of combination in (3.11) are given by

$$
k c \rightleftarrows[k R(c), k L(c), k W(c)], \quad k \varepsilon K,
$$

(3.12) $c_{1}+c_{2} \rightleftarrows\left[R\left(c_{1}\right)+R\left(c_{2}\right), L\left(c_{1}\right)+L\left(c_{2}\right), W\left(c_{1}\right)+W\left(c_{2}\right)\right]$,

$$
c_{1} c_{2} \rightleftarrows\left[R\left(c_{1}\right) L\left(c_{2}\right), L\left(c_{2}\right) L\left(c_{1}\right), R_{22}\left(c_{2}\right) W\left(c_{1}\right)+L_{22}\left(c_{1}\right) W\left(c_{2}\right)+\left\{\gamma_{1}, \gamma_{2}\right\}\right] .
$$

Here $\left\{\gamma_{1}, \gamma_{2}\right\}=\left(d_{1}, \cdots, d_{q}\right)^{\prime}$ is determined by

$$
\bar{\gamma}_{1} \bar{\gamma}_{2}=\overline{\gamma_{1} \gamma_{2}}+r\left(\gamma_{1}, \gamma_{2}\right) ; \quad r\left(\gamma_{1}, \gamma_{2}\right)=\sum_{k=1}^{q} d_{k} z_{k} \varepsilon \Re^{t}
$$

Proof. It is easily seen that (3.11) yields a one-to-one correspondence between the elements of $\mathfrak{A}$ and the symbols $[R(c), L(c), W(c)]$. For (3.8) expresses $c$ uniquely as the sum $\bar{\gamma}+r$ and $\bar{\gamma} \rightleftarrows \gamma, \gamma \rightleftarrows[R(c), L(c)], r \rightleftarrows W(c)$. It remains to show that the rules of combination (3.12) are in accord with this correspondence. For the sum and scalar product this is evident. For the product

$$
\begin{aligned}
c_{1} & =\bar{\gamma}_{1}+r_{1}, \quad c_{2}=\bar{\gamma}_{2}+r_{2}, \\
c_{1} c_{2} & =\bar{\gamma}_{1} \bar{\gamma}_{2}+\bar{\gamma}_{1} r_{2}+r_{1} \bar{\gamma}_{2}=\overline{\gamma_{1} \gamma_{2}}+\bar{\gamma}_{1} r_{2}+r_{1} \bar{\gamma}_{2}+r\left(\gamma_{1}, \gamma_{2}\right),
\end{aligned}
$$

where $\overline{\gamma_{1} \gamma_{2}} \rightleftarrows\left[R\left(c_{1}\right) R\left(c_{2}\right), L\left(c_{2}\right) L\left(c_{1}\right)\right]$ from (3.5) and $\bar{\gamma}_{1} r_{2} \rightleftarrows L_{22}\left(c_{1}\right) W\left(c_{2}\right), r_{1} \bar{\gamma}_{2}$ $\rightleftarrows R_{22}\left(c_{2}\right) W\left(c_{1}\right)$ by (3.10.3) and $r\left(\gamma_{1}, \gamma_{2}\right) \rightleftarrows\left\{\gamma_{1}, \gamma_{2}\right\}$ by (3.13). The element 
$r\left(\gamma_{1}, \gamma_{2}\right)$ will be called the remnant of the product $\bar{\gamma}_{1}, \bar{\gamma}_{2}$. Here $r_{1} r_{2}=0$ since $\left(\Re^{t}\right)^{2}=0$ because $\left(\Re^{t}\right)^{2} \subset \Re^{t} \Re=0$.

THEOREM 3.3. The remnants $r(x, y)$ of (3.13) satisfy the following relations:

$$
\begin{aligned}
r(x+y, z) & =r(x, z)+r(y, z), \\
r(x, y+z) & =r(x, y)+r(x, z), \\
r(k x, y) & =r(x, k y)=k r(x, y), \\
\bar{x} \bar{y} r(u, v) & =\bar{x} \bar{y} r(u, v), \\
r(u, v) \bar{x} \bar{y} & =r(u, v) \bar{x} \bar{y}, \\
\bar{x} r(y, z)+r(x, y z) & =r(x y, z)+r(x, y) \bar{z} .
\end{aligned}
$$

From the definition of the representative $\bar{\gamma}$ in (3.7) it follows immediately that

$$
\bar{x} \bar{y}=\bar{x}+\bar{y}, \quad \bar{k} \bar{x}=k \bar{x},
$$

whence the first three relations are easily derived. For the fourth relation, $\bar{x} \bar{y} r(u, v)=(\overline{x y}+r(x, y)) r(u, v)=\overline{x y} r(u, v)$ since $\left(\Re^{t}\right)^{2}=0$. The fifth relation may be derived in the same way. The last and perhaps the most important relation is obtained by multiplying out $\bar{x}(\bar{y} \bar{z})=(\bar{x} \bar{y}) \bar{z}$. In constructing a bound algebra with a given radical it is this last relation which is most difficult to satisfy.

If the representatives $\bar{u}_{1}, \cdots, \bar{u}_{m}$ are replaced by representatives $\overline{\bar{u}}_{1}, \cdots, \overline{\bar{u}}_{m}$ in the same classes of $\mathfrak{A}$ modulo $\mathfrak{R}^{t}$, then

$$
\begin{aligned}
\overline{\bar{u}}_{i} & =\bar{u}_{i}+\alpha\left(u_{i}\right), \quad i=1, \cdots, m, \\
\overline{\bar{x}} & =\bar{x}+\alpha(x),
\end{aligned}
$$

where $\alpha(x)$ satisfies the linearity conditions

$$
\alpha(x+y)=\alpha(x)+\alpha(y), \quad \alpha(k x)=k \alpha(x),
$$

$k \boldsymbol{\varepsilon} K$.

THEOREM 3.4. If the representatives of the classes of $\mathfrak{A}$ modulo $\mathfrak{R}^{t}$ are changed by the rule (3.17), then the remnants $r(x, y)$ are changed by the rule

$$
r^{\prime}(x, y)=r(x, y)+\bar{x} \alpha(y)+\alpha(x) \bar{y}-\alpha(x y) .
$$

Proof. $\overline{\bar{x}} \overline{\bar{y}}=(\bar{x}+\alpha(x))(\bar{y}+\alpha(y))$ or $\overline{\overline{x y}}+r^{\prime}(x, y)=\bar{x} \bar{y}+\bar{x} \alpha(y)+\alpha(x) \bar{y}$ or $\overline{x y}+\alpha(x y)+r^{\prime}(x, y)=\overline{x y}+r(x, y)+\bar{x} \alpha(y)+\alpha(x) \bar{y}$, whence (3.19) follows.

THEOREM 3.5. If $e_{1}, \cdots, e_{n}$ are orthogonal idempotents in $\mathfrak{A} / \Re^{t}$, then the representatives $\bar{e}_{1}, \cdots, \bar{e}_{n}$ may be chosen as orthogonal idempotents.

The proof of this theorem exactly parallels the proof of Theorem 1 on page 16 of Deuring's Algebren. 
TheOREM 3.6. In a bound algebra $\mathfrak{A}$ there are subalgebras $\mathfrak{A}_{l}, \mathfrak{A}_{r}, \mathfrak{A}_{d}$ such that $\mathfrak{A}=\mathfrak{A}_{l}+\mathfrak{A}_{r}+\left(\mathfrak{A}_{d} \cup \mathfrak{R}\right)$ with the following properties:

1. $\mathfrak{A}_{l}, \mathfrak{A}_{r}, \mathfrak{A}_{d}$ have units and are orthogonal.

2. $\mathfrak{A}_{l}$ and $\mathfrak{A}_{r}$ are semisimple and $\mathfrak{R} \mathfrak{A}_{l}=0, \mathfrak{A}_{r} \mathfrak{R}=0$.

3. $\mathfrak{A} / \mathfrak{R}$ is the direct sum of three semisimple algebras isomorphic to $\mathfrak{A}_{l}, \mathfrak{A}_{r}$, $\mathfrak{A}_{d} /\left(\mathfrak{A}_{d} \cap \mathfrak{R}\right)$.

Proof. $\Re^{r}$ and $\Re^{l}$ are both two-sided ideals and $\mathfrak{A}$ itself may be considered a two-sided ideal, whence by Theorem 2.1

$$
\begin{aligned}
\Re^{r}=\left(e_{1}\right)_{r}+\mathfrak{r}_{1}, & \Re^{r}=\left(e_{1}\right)_{l}+\mathfrak{r}_{2}, & \Re^{l}=\left(e_{2}\right)_{r}+\mathfrak{r}_{3}, \\
\Re^{l}=\left(e_{2}\right)_{l}+\mathfrak{r}_{4}, & \mathfrak{A}=(\epsilon)_{l}+\mathfrak{r}_{5}, & \mathfrak{A}=(e)_{l}+\mathfrak{r}_{6},
\end{aligned}
$$

with relations on the r's as given in Theorem 2.1.

Put $e_{3}=e-e_{1}-e_{2}$. Then it may be shown that $e_{i} e_{j}, i \neq j$, is a two-sided annihilator of $\Re$ and that consequently the images of $e_{1}, e_{2}$, and $e_{3}$ in $\mathfrak{A} / \Re^{t}$ are orthogonal idempotents. By Theorem 3.5 there exist orthogonal idempotents $e_{1}^{\prime}, e_{2}^{\prime}$, and $e_{3}^{\prime}$ in the classes of $e_{1}, e_{2}$, and $e_{3}$ modulo $\Re^{t}$. Just as in the proof of Theorem 2.1 it may be shown that $e_{1}^{\prime}, e_{2}^{\prime}$, and $e^{\prime}=e_{1}^{\prime}+e_{2}^{\prime}+e_{3}^{\prime}$ may be used in the representation of the ideals in (3.20). Stated formally:

Lемма. Without loss of generality it may be assumed that the idempotents of (3.20) satisfy the following relations:

$$
e e_{1}=e_{1} e=e_{1}, \quad e e_{2}=e_{2} e=e_{2}, \quad e_{1} e_{2}=e_{2} e_{1}=0 .
$$

Now put $\mathfrak{A}_{l}=e_{1} \mathfrak{A} e_{1}, \mathfrak{A}_{r}=e_{2} \mathfrak{A} e_{2}$, and $\mathfrak{A}_{d}=e_{3} \mathfrak{A} e_{3}$. Here $e_{1}, e_{2}$, and $e_{3}$ are the units of $\mathfrak{A}_{l}, \mathfrak{A}_{r}$, and $\mathfrak{A}_{d}$ respectively, and the orthogonality of these algebras is an immediate consequence of the orthogonality of these idempotents. This is the first property mentioned in the theorem.

To prove the decomposition

$$
\mathfrak{U}=\mathfrak{A}_{l}+\mathfrak{A}_{r}+\left(\mathfrak{\Re}_{d} \cup \mathfrak{R}\right),
$$

take any $x$ of $\mathfrak{A}$. From the relations (3.20) $x=e w+t$ where $t \varepsilon \mathfrak{R}, e t=0$. Here $e x=e w, x=e x+t$. Also $e x=u e+s$ with $s \varepsilon \Re, s e=0$ and so $e x e=u e, e x=e x e+s$, $x=e x e+s+t=e x e+\rho$ with $\rho \varepsilon \Re$. Hence $x=\left(e_{1}+e_{2}+e_{3}\right) x\left(e_{1}+e_{2}+e_{3}\right)+\rho$ $=e_{1} x e_{1}+e_{2} x e_{2}+e_{3} x e_{3}+\rho^{*}=x_{l}+x_{r}+\left(x_{d}+\rho^{*}\right)$. Here $\rho^{*}=\sum_{i \neq j} e_{i} x e_{j}+\rho \varepsilon \Re$ since $e_{i} x e_{j}$ is in the radical. For $e_{1} x e_{j} \varepsilon \Re^{r}=\left(e_{1}\right)_{l}+\mathfrak{r}_{2}, e_{1} x e_{j}=u e_{1}+t$ where $t \varepsilon \Re$, $t e_{1}=0$. Hence $0=e_{1} x e_{j} e_{1}=u e_{1}$ and $e_{1} x e_{j}=t \varepsilon \Re$. A similar argument holds for all the $e_{i} x e_{j}, j \neq i$. To show that the sum $x=x_{l}+r_{r}+\left(x_{d}+\rho^{*}\right)$ is unique it is enough to show that

$$
0=x_{l}+x_{r}+\left(x_{d}+\rho^{*}\right)
$$

implies that $x_{l}, x_{r}$, and $x_{d}+\rho^{*}$ all vanish. For $0=x_{l} e_{1}+x_{r} e_{1}+\left(x_{d}+\rho^{*}\right) e_{1}$ and here $x_{r} e_{1}=x_{r} e_{2} e_{1}=0, x_{d} e_{1}=x_{d} e_{3} e_{1}=0$, and since $\rho^{*} \varepsilon \Re, \rho^{*} e_{1}=0$. Hence $x_{l}=x_{l} e_{1}$ 
$=0$. Then $0=e_{2} x_{r}+e_{2}\left(x_{d}+\rho^{*}\right)$ and $e_{2} x_{d}=0, e_{2} \rho^{*}=0$, and so $x_{r}=e_{2} x_{r}=0$. Now as $x_{l}=0, x_{r}=0$, then from (3.23) $x_{d}+\rho^{*}=0$. This proves the decomposition (3.22).

For the second property, since $\mathfrak{A}_{r}=e_{2} \mathfrak{A} e_{2}$ and $e_{2} \mathfrak{R}=0$ it follows that $\mathfrak{A}_{r} \Re=0$. Similarly $\mathfrak{R} \mathfrak{A}_{l}=0$.

It remains to show that $\mathfrak{A}_{r}$ and $\mathfrak{A}_{l}$ are semisimple. If $\mathfrak{A}_{r}$ contained a nilpotent ideal $\mathfrak{r}$, then

$$
\mathfrak{r} \mathfrak{A} \subset \mathfrak{r} \mathfrak{A}_{r}+\mathfrak{r} \mathfrak{R}=\mathfrak{r}+\mathfrak{r} \mathfrak{R}
$$

by the decomposition (3.22) and the orthogonality of $\mathfrak{A}_{l}, \mathfrak{A}_{r}$, and $\mathfrak{A}_{d}$. Here $\mathfrak{r}+\mathfrak{r} \Re$ would be a nilpotent ideal in $\mathfrak{A}$ and hence contained in $\Re$, whence $\mathfrak{r}$ would be contained in $\Re$. But $\mathfrak{A}_{r}$ cannot contain any elements of $\Re$ since $e_{2}$, the unit of $\mathfrak{A}_{r}$, is a left annihilator of $\Re$. In the same way it may be shown that $\mathfrak{A}_{l}$ is semisimple.

Applying the homomorphism $\mathfrak{A} \rightarrow \mathfrak{A} / \mathfrak{R}$ to the decomposition (3.22) we have

$$
\mathfrak{A} / \mathfrak{\Re}=\tilde{\mathfrak{A}}_{l} \oplus \tilde{\mathfrak{A}}_{r} \oplus \tilde{\mathfrak{A}}_{d},
$$

$\tilde{\mathfrak{A}}_{i}$ being the image of $\mathfrak{A}_{i}$. The sum is direct since the $e_{i}$ and a fortiori their images are orthogonal. Since $\mathfrak{A}_{l}$ and $\mathfrak{A}_{r}$ are semisimple they must be iso-

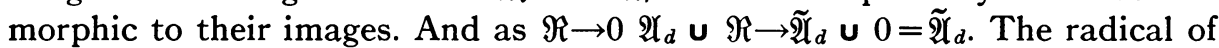
$\mathfrak{A}_{d}$ is $\mathfrak{A}_{d} \cap \mathfrak{R}$ and so $\tilde{\mathfrak{A}}_{d}=\mathfrak{A}_{d} /\left(\mathfrak{A}_{d} \cap \mathfrak{R}\right)$.

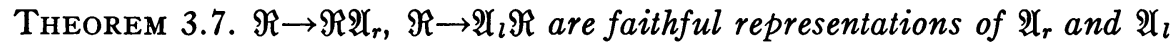
respectively. Neither of the mappings $\Re \rightarrow \Re \mathfrak{A}_{d}$ and $\Re \rightarrow \mathfrak{A}_{d} \Re$ maps onto zero any element of $\mathfrak{A}_{d}$ not in $\mathfrak{R}$. Hence $\mathfrak{R} \rightarrow \mathfrak{R} \mathfrak{A}_{d}$ is a faithful representation of $\mathfrak{A}_{d} / \mathfrak{b}_{1}$ and $\Re \rightarrow \mathfrak{A}_{d} \mathfrak{R}$ is a faithful representation of $\mathfrak{A}_{d} / \mathfrak{b}_{2}\left({ }^{4}\right)$ where $\mathfrak{b}_{1}, \mathfrak{b}_{2} \subset \Re, \mathfrak{b}_{1}^{2}=0$, $\mathfrak{b}_{2}^{2}=0$.

Suppose the mapping $\Re \rightarrow \Re \mathfrak{A}_{r}$ represents some $z$ of $\mathfrak{A}_{r}$ as 0 . Then $\Re z=0$, but $\mathfrak{A}_{r} \Re=0$, and so $z \Re=0$. Hence as a two-sided annihilator of $\Re, z$ belongs to $\Re$. But by the preceding theorem $\mathfrak{A}_{r}$ contains no elements of $\Re$. Hence $z=0$ and $\Re \rightarrow \Re \mathfrak{A}_{\text {r }}$ is a faithful representation. Similarly $\Re \rightarrow \mathfrak{A}_{l} \Re$ is a faithful representation.

If $\Re \rightarrow \Re \mathfrak{A}_{d}$ represents a $z \varepsilon \mathfrak{A}_{d}$ by zero, then $\mathfrak{R} z=0, z \varepsilon \Re^{r}=\left(e_{1}\right)_{r}+\mathfrak{r}_{1}$ $=\left(e_{1}\right)_{l}+\mathfrak{r}_{2}$. For any $w \varepsilon \Re^{r}, w=e_{1} w e_{1}+t$ with $t \varepsilon \Re, e_{1} w e_{1} \varepsilon \mathfrak{A}_{l}$, and so for $z \varepsilon \mathfrak{R}^{r}, z \varepsilon \mathfrak{A}_{d}, z=t \varepsilon \mathfrak{R}$. Hence the only elements of $\mathfrak{A}_{d}$ represented by zero in $\Re \rightarrow \Re \mathfrak{A}_{d}$ are elements of $\Re$. Those elements mapped on to zero form a twosided ideal $\mathfrak{b}_{1}$ in $\Re$, and since $\Re \mathfrak{b}_{1}=0$ a fortiori $\mathfrak{b}_{1}^{2}=0$. Similarly we may treat the representation $\Re \rightarrow \mathfrak{A}_{d} \Re$.

THEOREM 3.8. If a radical $\Re$ is of order $s$, then an algebra $\mathfrak{A}$ bound to $\Re$ is at most of order $s^{2}+s+1$.

(4) These two algebras may be different. See Example 2 in $\$ 4$. 
Proof. Consider the decomposition (3.22) of $\mathfrak{A}$ and the representation (3.11) of $\mathfrak{A}$. Since neither $\mathfrak{A}_{l}$ nor $\mathfrak{A}_{r}$ contains any elements of $\mathfrak{R}^{t}$, we may choose their elements as representatives of their classes modulo $\Re^{t}$ in (3.11) and hence

$$
\begin{array}{ll}
c \rightleftarrows[0, L(c), 0], & c \varepsilon \mathfrak{A}_{l}, \\
c \rightleftarrows[R(c), 0,0], & c \varepsilon \mathfrak{A}_{r} ;
\end{array}
$$

whence $\mathfrak{A}_{l}$ may be called the left represented subalgebra, $\mathfrak{A}_{r}$ the right represented subalgebra. In the sense of Theorem $3.7 \mathfrak{A}_{d}$ may be called the doubly represented subalgebra.

Now we appeal to the theorems on fully reducible matric algebras as they appear in Weyl's The Classical Groups, Their Invariants and Representations, chap. 3 . As $\mathfrak{A}_{l}$ is semisimple, the representation $\Re \rightarrow \mathfrak{A}_{l} \mathfrak{R}$ is fully reducible, the irreducible components corresponding to the simple algebras whose direct sum is $\mathfrak{A}_{l}$. If $\mathfrak{A}_{l}=\mathfrak{A}_{1} \oplus \cdots \oplus \mathfrak{A}_{n}$ where the $\mathfrak{A}_{i}$ are simple, then the representation breaks up into blocks of degrees $g_{1}, \cdots, g_{n}, g_{n+1}=s-\left(g_{1}+g_{2}+\cdots+g_{n}\right)$, the $i$ th block containing a certain number of equivalent irreducible representations of $\mathfrak{A}_{i}$, and the last vanishing. The commutator algebra of $\mathfrak{A}_{l}$ will break up into blocks $B_{1}, \cdots, B_{n}, B_{n+1}$ where $B_{i}$ is the commutator algebra of the $i$ th block, $i=1, \cdots, n$, and $B_{n+1}$ is the complete $g_{n+1}^{2}$ matric algebra. From Weyl, page 93 , the orders $h_{i}$ and $h_{i}^{\prime}$ of $\mathfrak{A}_{i}$ and $B_{i}$ respectively satisfy $h_{i} h_{i}^{\prime}=g_{i}^{2}$ for $i=1, \cdots, n$ and $h_{n+1}^{\prime}=g_{n+1}^{2}$.

Now every element of $\mathfrak{A}_{r}$ and every element of $\mathfrak{A}_{d}$ not in $\Re$ has a proper representation $c \rightleftarrows R(c)$ from Theorem 3.7, and by Theorem $3.1 R(c)$ must be a subalgebra of the commutator algebra of $L(c)$. Hence the order of $\mathfrak{A}$ does not exceed the order of $\mathfrak{A}_{l}$ plus the order of the commutator of $\mathfrak{A}_{l}$ plus the order of $\Re$. Hence the order of $\mathfrak{A}$ is at most

$$
\sum_{i=1}^{n}\left(h_{i}+h_{i}^{\prime}\right)+g_{n+1}^{2}+s=\sum_{i=1}^{n}\left(h_{i}+g_{i}^{2} / h_{i}\right)+g_{n+1}^{2}+s .
$$

The $g$ 's and $h$ 's are positive in tegers ( $g_{n+1}$ might be zero) and the sum of the $g$ 's is $s$. Here it is very easy to show that

$$
\sum_{i=1}^{n}\left(h_{i}+g_{i}^{2} / h_{i}\right)+g_{n+1}^{2} \leqq s^{2}+s+1
$$

and that equality holds only when $n=1, g_{1}=s$, and $h_{1}=1$ or $s^{2}$. This proves the theorem. This result is the best possible since we could have an algebra with $\mathfrak{R}^{2}=0, \mathfrak{A}_{l}$ the complete $s^{2}$ matric algebra and $\mathfrak{A}_{r}$ the scalar algebra of order 1 . On the other hand, when $\Re^{2} \neq 0$ the order of $\mathfrak{A}$ must be less than $s^{2}+s+1$ and it should be possible to obtain various improvements on this theorem.

4. Construction of bound algebras. In constructing all algebras bound to 
a given radical $\Re$, we turn to representation (3.11), remembering that from Theorem 3.6 we need determine only $\mathfrak{A}_{r}, \mathfrak{A}_{l}, \mathfrak{A}_{d}$ separately. When $\mathfrak{A}_{d}$ is void, equations (3.25) make the construction of the algebra relatively easy. Any two semisimple matric algebras which permute with each other and the representations of elements of $\Re$ may be considered the right and left represented subalgebras of an algebra bound to $\Re$. The construction of algebras in which $\mathfrak{A}_{d}$ is not void offers many more difficulties. In the first place $\mathfrak{A}_{d}$ is not usually semisimple and its right and left representations may be different algebras. Moreover the faithful representation of $\mathfrak{A}_{d}$ in (3.11) may involve remnants which must be chosen to satisfy equations (3.15).

THEOREM 4.1. Given a nilpotent algebra $\Re$ of order $s$, let the right representation of $r \varepsilon \Re$ on a basis of $\Re$ be $r \rightarrow R(r)$ and the left representation be $r \rightarrow L(r)$. If, in the homomorphism $\Re \rightarrow \Re / \Re^{t}, r \rightarrow \rho$, then $\rho \rightleftarrows[R(r), L(r)]$ is a faithful representation of $\Re / \Re^{t}$. Suppose $(1) c \rightleftarrows[R(c), L(c)]$ is a right-left representation of an $s$ by $s$ matric algebra $\mathfrak{H}^{\prime}$ whose radical is $\Re / \Re^{t}$ and that every $R\left(c_{1}\right)$ permutes with every $L\left(c_{2}\right)$; (2) remnants $r(x, y)$ are chosen from $\Re^{t}$ for every pair $x, y$ of elements of $\mathfrak{A}^{\prime}$ such that equations (3.15) are satisfied; and (3) the remnants $r\left(\rho_{i}, \rho_{j}\right)$ are such that $\bar{\rho}_{i}$ may be considered representatives of classes of $\Re$ modulo $\Re^{t}$. Then (3.11) yields a faithful representation of an algebra bound to $\Re$, the rules of combination being given by (3.12) and (3.13).

Theorem 3.2 shows that every bound algebra has a representation (3.11). This theorem shows that conversely the symbols (3.11) define an algebra bound to $\Re$ providing that certain conditions are satisfied. The proof is direct though a little tedious and will only be sketched here. It must be shown that the rules (3.12) and (3.13) actually define an associative algebra and it is here that we need equations (3.15) and the permutability of $R\left(c_{1}\right)$ and $L\left(c_{2}\right)$. Moreover conditions 1 and 2 assure us that the radical of this algebra is $\Re$, and neither more nor less. That $\mathfrak{A}$ is bound to $\Re$ is an immediate consequence of the fact that the elements of $\mathfrak{A}$ are properly represented on $\Re$ apart from $\Re^{t} \subset \Re$.

In practise the following theorem is of use:

THEOREM 4.2. $c \rightarrow R(c)$ is a faithful representation of $\mathfrak{A} / \Re^{r}$, and the radical of $[R(c)]$ is the right representation of $\Re . \mathfrak{A} / \Re^{r}$ modulo its radical is isomorphic to $\tilde{\mathfrak{A}}_{r} \oplus \tilde{\mathfrak{A}}_{d}$. Similarly the radical of $[L(c)]$ is the left representation of $\Re$, and $[L(c)]$ modulo its radical is isomorphic to $\tilde{\mathfrak{A}}_{l} \oplus \tilde{\mathfrak{A}}_{d}$.

Proof. The matric algebra $[R(c)]$ is $\mathfrak{A} / \Re^{r}$ since it is a homomorphic image of $\mathfrak{A}$ in which the elements mapped onto zero are those of $\Re^{r}$. Suppose $T$ is its radical. The elements of $\mathfrak{A}$ mapped onto $T$ and zero form a two-sided ideal $K$, and include $\mathfrak{A}_{l}$ and $\Re$. Since $T^{u}=0, K^{u} \subset \Re^{r}$. From Theorem $2.1 K=(e)_{r}+\mathfrak{r}$ with $\mathfrak{r} \subset \Re$. Now $e \varepsilon K^{u} \subset \Re^{r}$. Hence $(e)_{r}$ is represented by zero and $T$ is the representation of $\mathfrak{r}$ alone. Hence $T$ is the image of $\Re$, and the only elements 
of $\mathfrak{A}$ mapped onto $T$ and zero are $\mathfrak{A}_{l}+\mathfrak{R}$. From the decomposition (3.22) $\mathfrak{A} / \mathfrak{R}^{r}$ modulo its radical is isomorphic to $\tilde{\mathfrak{A}}_{r} \oplus \tilde{\mathfrak{A}}_{d}$. The proof for $[L(c)]$ is similar.

EXAMPLE 1. $\Re$ is cyclic: $\Re=\left(x, \cdots, x^{n-1}\right)$, where $x^{n}=0$.

Case 1. $\mathfrak{A}=\Re$.

Case 2. $\mathfrak{A} \neq \mathfrak{R}$ and $\mathfrak{A}=\mathfrak{R}^{l}$ u $\mathfrak{R}$. Here $\mathfrak{A}=\left(e_{2}\right)_{r}+\mathrm{r}$. Now $x e_{2}=a_{1} x+a_{2} x^{2}+\cdots$ $+a_{n-1} x^{n-1}$ and since $e_{2}^{2}=e_{2}, x^{n}=0$, it follows that $x e_{2}=0$ or $x$. Similarly $e_{2} x=0$ or $x$. As $\mathfrak{A}=\Re^{l} \cup \Re, e_{2} x=0$. Now if also $x e_{2}=0$, then $e_{2} \varepsilon \Re^{t} \subset \Re$, which is impossible. Hence $x e_{2}=x$. An arbitrary $y \varepsilon \mathfrak{A}$ is of the form $y=e_{2} y e_{2}+t$ with $t \varepsilon \Re$. Let $x e_{2} y e_{2}=b_{1} x+\cdots+b_{n-1} x^{n-1}$. Then put $v=e_{2} y e_{2}-b_{1} e_{2}$. Here $v x=0$, $x v=b_{2} x^{2}+\cdots+b_{n-1} x^{n-1}$. From this $(v)_{r}^{n} \subset \Re^{r}$, and, since $(v)_{r}^{n} \subset \Re^{l},(v)_{r}^{n} \subset \Re^{t}$ $\subset \Re$, whence $(v)_{r}$ is a nilpotent ideal and $v \varepsilon \Re$. Hence $v=e_{2} v e_{2} \varepsilon e_{2} \Re e_{2}=0$ and $v=0$. Hence $y=b_{1} e_{2}+t$ where $t \varepsilon \Re$. Here $\mathfrak{A}=\left(e_{2}, x, \cdots, x^{n-1}\right)$ with $e_{2} x=0$, $x e_{2}=x$.

Case 3. $\mathfrak{A} \neq \mathfrak{R}^{\imath} \cup \mathfrak{R}$. Here $\mathfrak{A}=(e)_{l}+\mathfrak{r}$, and, arguing as above, we conclude $e x=x, x e=0$ or $x$ while $\mathfrak{A}=(e)+\Re^{l} \cup \mathfrak{R}$. We distinguish according as $x e=0$ or $x$, and as $\Re^{l} \cup \Re=\Re$ or $\left(e_{2}, \Re\right)$.

Case 3.1. $\mathfrak{A}=\left(e_{1}, e_{2}, \mathfrak{R}\right)$ :

$$
\begin{aligned}
e_{1}^{2} & =e_{1}, & e_{2}^{2} & =e_{2}, & e_{1} e_{2} & =e_{2} e_{1}=0, \\
e_{1} x & =x, & x e_{1} & =0, & e_{2} x & =0, \quad x e_{2}=x .
\end{aligned}
$$

Case 3.2. $\mathfrak{A}=\left(e_{1}, \mathfrak{R}\right)$ :

$$
e_{1}^{2}=e_{1} . \quad e_{1} x=x, \quad x e_{1}=0 .
$$

Case 3.3. $\mathfrak{A}=(e, \Re)$ :

$$
e^{2}=e, \quad e x=x e=x .
$$

Thus in all five cases and for any radical five similar bound algebras will exist. Let $\Re$ be any nilpotent algebra and $e_{1}$ and $e_{2}$ two orthogonal idempotents. For any $r \varepsilon \Re$ let $e_{1} r=r, r e_{1}=0, e_{2} r=0, r e_{2}=r$. Then we might have (1) $\mathfrak{A}=\mathfrak{R},(2) \mathfrak{A}=\left(e_{1}, \mathfrak{R}\right)$, (3) $\mathfrak{A}=\left(e_{2}, \mathfrak{R}\right)$, (4) $\mathfrak{A}=\left(e_{1}+e_{2}, \mathfrak{R}\right)$, or $(5) \mathfrak{A}=\left(e_{1}, e_{2}, \mathfrak{R}\right)$.

EXAMPLE 2. Let $\Re$ have a basis $x_{1}, x_{2}, x_{3}, x_{4}$ where $x_{1}^{2}=x_{3}, x_{1} x_{2}=x_{4}, x_{1} x_{3}=0$, $x_{1} x_{4}=0$ and $x_{i}^{2}=x_{i} x_{j}=0$ for $i \neq 1$.

Here

$$
x_{1} \rightarrow\left[\left(\begin{array}{llll}
0 & 0 & 1 & 0 \\
0 & 0 & 0 & 0 \\
0 & 0 & 0 & 0 \\
0 & 0 & 0 & 0
\end{array}\right),\left(\begin{array}{llll}
0 & 0 & 1 & 0 \\
0 & 0 & 0 & 1 \\
0 & 0 & 0 & 0 \\
0 & 0 & 0 & 0
\end{array}\right)\right]
$$




$$
x_{2} \rightarrow\left[\left(\begin{array}{llll}
0 & 0 & 0 & 1 \\
0 & 0 & 0 & 0 \\
0 & 0 & 0 & 0 \\
0 & 0 & 0 & 0
\end{array}\right),\left(\begin{array}{llll}
0 & 0 & 0 & 0 \\
0 & 0 & 0 & 0 \\
0 & 0 & 0 & 0 \\
0 & 0 & 0 & 0
\end{array}\right)\right]
$$

while $x_{3}$ and $x_{4}$ are represented by 0 on both sides. Note that the radicals of $[R(c)]$ and $[L(c)]$ are not of the same order. By Theorem 3.1 the elements of $[R(c)]$ are of the type

$$
\left(\begin{array}{llll}
a_{1} & b_{1} & e_{1} & f_{1} \\
c_{1} & d_{1} & g_{1} & h_{1} \\
0 & 0 & a_{1} & b_{1} \\
0 & 0 & c_{1} & d_{1}
\end{array}\right)
$$

and those of $[L(c)]$ of the type

$$
\left(\begin{array}{llll}
a_{2} & b_{2} & e_{2} & f_{2} \\
0 & d_{2} & g_{2} & h_{2} \\
0 & 0 & a_{2} & 0 \\
0 & 0 & 0 & a_{2}
\end{array}\right)
$$

Here aside from the matrix corresponding to $x_{1},[L(c)]$ can contain the unit matrix I, an idempotent

$$
E=\left(\begin{array}{cccc}
0 & b_{2} & b_{2} g_{2} & b_{2} h_{2} \\
0 & 1 & g_{2} & h_{2} \\
0 & 0 & 0 & 0 \\
0 & 0 & 0 & 0
\end{array}\right)
$$

the idempotent $I-E$, or only one of these. If we use the automorphism

$$
\begin{aligned}
& x_{1} \rightleftarrows x_{1}-b_{2} x_{2}-b_{2} g_{2} x_{3}-b_{2} h_{2} x_{4}, \\
& x_{2} \rightleftarrows \\
& x_{3} \rightleftarrows \\
& x_{2} \rightleftarrows g_{2} x_{3}+h_{2} x_{4}, \\
& x_{4} \rightleftarrows
\end{aligned}
$$

$E$ takes the simple form

$$
E=\left(\begin{array}{llll}
0 & 0 & 0 & 0 \\
0 & 1 & 0 & 0 \\
0 & 0 & 0 & 0 \\
0 & 0 & 0 & 0
\end{array}\right)
$$


In case $E$ (or $I-E$ ) actually occurs in $[L(c)]$, then by Theorem $3.1[R(c)]$ is further restricted to matrices of the form

$$
\left(\begin{array}{llll}
a_{1} & 0 & e_{1} & f_{1} \\
0 & d_{1} & 0 & 0 \\
0 & 0 & a_{1} & 0 \\
0 & 0 & 0 & d_{1}
\end{array}\right)
$$

Here write

$$
F=\left(\begin{array}{llll}
1 & 0 & 0 & 0 \\
0 & 0 & 0 & 0 \\
0 & 0 & 1 & 0 \\
0 & 0 & 0 & 0
\end{array}\right)
$$

From here on it is fairly simple to enumerate the possible algebras bound to $\Re$.

Case I. $\mathfrak{A}_{d}$ is void.

Here $\mathfrak{A}=\mathfrak{A}_{r}+\mathfrak{A}_{l}+\mathfrak{R}$ and $\mathfrak{A}_{r}$ and $\mathfrak{A}_{l}$ are faithfully represented without remnants.

(a) $\mathfrak{A}_{l}$ is void or $I$. Then $\mathfrak{A}_{r}$ is void or any semisimple matric algebra of the permissible form. These can be of order 1,2 , or 4 .

(b) $\mathfrak{A}_{l}$ contains $E, I-E$, or both. Then $\mathfrak{A}_{r}$ is void or contains $F, I-F$ or both.

Case II. $\mathfrak{A}_{d}$ contains only one element independent of $\Re$. This element can be taken as an idempotent $e$.

(a) $e$ is a left unit of $\mathfrak{A}$.

$\mathfrak{A}_{l}$ must be void. If $e$ is a right unit, then $\mathfrak{A}_{r}$ is void, and $\mathfrak{A}=\mathfrak{A}_{d}=(1, \mathfrak{R})$. We may also have, using automorphisms to simplify the form of the matrices, $e \rightleftarrows[F, I, 0]$. Here $\mathfrak{A}_{d}=\left(e, x_{1}, x_{3}\right)$ and $\mathfrak{A}_{r}$ is void or has an idempotent $f \rightleftarrows[I-F, 0,0]$. It is also possible that $e \rightleftarrows[I-F, I, 0]$ and $\mathfrak{A}_{d}=\left(e, x_{2}, x_{4}\right)$ while $\mathfrak{A}_{r}$ is void or has an idempotent $f \rightleftarrows[F, 0,0]$.

(b) $e$ is a right unit of $\mathfrak{A}$.

The possibilities here are similar to those above.

(c) $e$ is neither a right nor a left unit of $\mathfrak{A}$.

Here $e$ has $E$ or $I-E$ as its left representation and $F$ or $I-F$ as its right representation while $\mathfrak{A}_{r}$ and $\mathfrak{A}_{l}$ are void or contain the idempotent $E, I-E$, $F, I-F$ not representing $e$.

Case III. $\mathfrak{A}_{d}$ contains two elements independent of $\Re$.

Here $\mathfrak{A}_{r}$ and $\mathfrak{A}_{l}$ are both void and $\mathfrak{A}=\mathfrak{A}_{d}$. $\mathfrak{A}$ has a unit. $\mathfrak{A}=(1, e, \mathfrak{R})$ where $1 \rightleftarrows[I, I, 0]$ and $e \rightleftarrows[F, E, 0]$ or $[F, I-E, 0]$ or $[I-F, E, 0]$ or $[I-F$, $I-E, 0]$.

Throughout this example, as a consequence of Theorem 3.5, all remnants may be taken as zero. 
EXAmple 3. $\Re=\left(x_{1}, x_{2}\right), \Re^{2}=0, K$ of characteristic 2 .

In a particular algebra bound to $\Re$,

$$
\begin{aligned}
& 1 \rightleftarrows[I, I, 0], \\
& y \rightleftarrows\left[\left(\begin{array}{ll}
0 & 1 \\
a & 0
\end{array}\right),\left(\begin{array}{ll}
0 & 1 \\
a & 0
\end{array}\right)\left(\begin{array}{l}
0 \\
0
\end{array}\right)\right],
\end{aligned}
$$

where $a$ is not a square in $K$. Here we may take $r(1,1)=r(1, y)=r(y, 1)=0$, but any value $r(y, y)=b x_{1}+c x_{2}$ will be permissible under equations (3.15), and since $K$ is of characteristic 2 a change of representative will not affect the remnants. This is a case in which the center of one of the simple algebras of $\mathfrak{X} / \mathfrak{R}$ is inseparable.

YALE UNIVERSITY, New Haven, Conn. 